\title{
Path Coefficient Analysis of Yield Components of Rice under Normal and Aerobic Conditions
}

\author{
Khushboo Chandra* and Nilanjaya
}

Dept. of Plant Breeding and Genetics, Dr.RPCAU, Pusa, Samastipur, Bihar (848 125), India

\section{Corresponding Author}

Khushboo Chandra

e-mail: cute.khushi6@gmail.com

\author{
Article History \\ Article ID: 3 C0110 \\ Received in $8^{\text {th }}$ September, 2017 \\ Received in revised form $17^{\text {th }}$ November, 2017 \\ Accepted in final form $3^{\text {rd }}$ December, 2017
}

\begin{abstract}
Selection for yield parameters is often difficult in crop breeding because yield is a complex inherited character controlled polygenically, contributed by several component traits. Twenty five genotypes of rice were evaluated under aerobic and normal conditions to study the path coefficient analysis based on fifteen morpho-physiological traits. Path analysis revealed at genotypic level that under aerobic and normal condition traits like days to $50 \%$ flowering $(0.487)$, no. of spikelets panicle ${ }^{-1}(0.572)$, number of tillers plant ${ }^{-1}(0.688)$, relative water content in flag leaf $(0.98)$ and maximum root length $(0.832,1.18)$ had highest positive direct effect whereas, proline accumulation in leaves(-0.31) had high negative direct effect under normal condition and these important plant traits must be taken into consideration when further breeding program for higher yield in rice under aerobic and normal conditions is to be planned. Characters like days to fifty percent flowering, relative water content, maximum root length and proline were noticed to exert high direct effects on grain yield plant ${ }^{-1}$. Panicle length showed positive association with number of spikelets panicle ${ }^{-1}, 1000$ grain weight and grain yield per plot, whereas it showed negative correlation with proline accumulation in leaves under both conditions. Hence, these traits may be helpful in selection of suitable parents for future breeding programmes. Grain yield per plot showed negative and non-significant association with days to fifty per cent flowering under control condition indicating early maturing genotypes should be preferred under water limited condition. It indicated that earliness enabled the cultivars to escape drought at the end of the growing season under water stress condition. High indirect effects of most of the traits were noticed mostly through panicle characters indicating importance of the trait as selection criteria in crop yield improvement programs.
\end{abstract}

Keywords: Path analysis, aerobic, Rice (Oryza sativa L), yield components

\section{Introduction}

Rice, a popularly known as Global grain, is a model crop, belonging to genus Oryza, family, Gramineae (Poaceae) and sub family Oryzoideae. The slogan "Rice is life" is most appropriate for India as this crop plays a vital role in our national food security and is a means of livelihood for millions of households. A Chinese proverb says, "Most precious things are not jade and pearls but rice grains". Rice and agriculture are still fundamental to the economic development of most of the Asian countries. In much of Asia, rice plays a central role in politics, society and culture, directly or indirectly employs more people than any other sector. India, it accounts for more than $40 \%$ of food. It is grown in 44.6 mha under 4 major ecosystems: irrigated ( $21 \mathrm{mha}$ ), rainfed lowland (14 million ha), rainfed upland ( $6 \mathrm{mha}$ ) and flood-prone (3 mha) with average annual of $96.4 \mathrm{mt}$ (NABARD, 2007-08). Grain yield is complex trait which is influenced by a number of contributing characters. Wright (1921) developed path coefficient analysis, a statistical device which is unique in partitioning the association into direct and indirect effects through other dependent variables. Unlike the correlation coefficient values which measure the extent of relationship, path coefficient (Wright, 1921; Dewey and Lu, 1959) measure the magnitude of direct and indirect effects of characters on complex dependent characters like yield and thus enable the breeders to judge best about the important component characters during selection. The ultimate aim of the path analysis is to accept descriptions of the correlation between the traits, based on a model of cause and effect relationship and to estimate the importance of the affecting traits on specific traits (Milligan et al., 1990). Path co-efficient also provide useful information regarding the direct and indirect effects of different yield component characters on grain yield and thus aid in the identification of effective selection criteria for effective yield improvement. This study was conducted to undertake comparison between direct and indirect effects of different traits on yield and to identify better combination as selection criteria for developing high yielding rice genotypes under normal and aerobic condition. A good knowledge of genetic resources might also help in identifying desirable genotypes for future hybridization program. 


\section{Materials and Methods}

The experimental material for the present study comprised of twenty five genotypes of rice suitable for aerobic and normal condition procured from Rajendra Agricultural University, Pusa laid in randomized block design (RBD) with three replications at the Field Experimentation Centre of Department of Plant Breeding and Genetics, Rajendra Agricultural University, Pusa Samastipur Bihar during kharif, 2014. Standard agronomic practices and plant protection measures were taken as per schedule. Each genotype was grown in a plot of $5 \times 1 \mathrm{~m}^{2}$ with the spacing $20 \times 15 \mathrm{~cm}^{2}$ row to row and plant to plant respectively. Observations were recorded for days to $50 \%$ flowering, days to physiological maturity, plant height $(\mathrm{cm})$, panicle length $(\mathrm{cm})$, number of spikelets panicle ${ }^{-1}$, number of tillers plant ${ }^{-1}, 1000$-grain weight $(\mathrm{g})$, relative water content (\%), maximum root length $(\mathrm{cm})$, Flag leaf area $\left(\mathrm{cm}^{2}\right)$, Harvest index (\%), Chlorophyll content (SPAD), Proline accumulation in leaves $\mathrm{g} \mathrm{g} \mathrm{g}^{-1}$ Dry Wt., Peroxidase activity in leaves unit $\mathrm{g}^{-1}$ fresh $\mathrm{Wt}$. and grain yield plot $^{-1}(\mathrm{~g})$ on five randomly selected plants from each entry for each replication for above mentioned traits excluding for days to flowering, days to physiological maturity and grain yield $\left(\mathrm{kg} \mathrm{plot}^{-1}\right)$ where whole plot data were considered.

\subsection{Relative water content in flag Leaf (\%)}

Relative water content (RWC) of the flag leaves was determined using the equation given by Barr and Weatherley (1962).

$$
\text { RWC }=\frac{\text { F.W.-D.W. }}{\text { T.W.-D.W. }} \times 100
$$

Where,

F.W.=Fresh Weight of flag leaf $(g)$

D.W.=Dry Weight of flag leaf $(\mathrm{g})$

T.W.=Turgid Weight of flag leaf (g)

\subsection{Flag leaf area $\left(\mathrm{cm}^{2}\right)$}

Flag leaf area was measured with the following function by Muller (1991).

Flag leaf area=Flag leaf length $\times$ Flag leaf width $\times 0.74$

\subsection{Harvest index (\%)}

The value of harvest index (HI) was calculated from the following formula (Donald, 1962).

$$
\begin{array}{r}
\text { Harvest index }=-100 \\
\text { Total biological yield (grain yield }+ \text { straw) }
\end{array}
$$

\subsection{Chlorophyll content (SPAD)}

Leaf chlorophyll content was recorded by measuring greenness of leaf using a portal chlorophyll meter (Monilta Camera Co. Ltd., Japan). SPAD readings were collected from the middle region of first fully opened leaf and it was averaged across each plot and expressed as SPAD reading per plant.

\subsection{Proline accumulation in leaves $\mu g g^{-1}$ Dry Wt.}

Proline Accumulation in Leaves was determined by the method developed by Bates et al. (1973). Peroxidase Activity in Leaves unit g Fresh Wt. The activity of peroxidase was determined by the method of Palmiano and Juliano (1973).

\section{Results and Discussion}

On perusal of Table 1, 2, 3 and 4 at genotypic level under normal condition high positive direct effect was exhibited by plant height, panicle length, number of tillers per plant, 1000 grain weight, relative water content, maximum root length whereas high negative direct effect was exhibited by days to physiological maturity, days to $50 \%$, number of spikelets panicle ${ }^{-1}$, flag leaf area, harvest index, chlorophyll content, proline accumulation in leaves and peroxidise activity in leaves. At phenotypic level positive direct effect was exhibited by days to $50 \%$ flowering, days to physiological maturity, plant height, panicle length, number of spikelets panicle ${ }^{-1}$, no. of tillers plant $^{-1}, 1000$ grain weight, relative water content, maximum root length, flag leaf area, whereas moderate negative direct effect was exhibited by harvest index, chlorophyll content, proline accumulation in leaves, peroxidase activity in leaves. These results were consistent with the findings of Agahi et al. (2007); Nagaraju et al. (2013) for grains per panicle, panicle plant $^{-1}$ and 1000-grain weight, Mustafa and Elsheikh (2007) and Patel et al. (2014) for days to 50\% flowering, Petchiammal and Kumar (2007) for panicle length, Ullah et al. (2011) for chlorophyll content. Hence, selection based on these characters would be more effective for yield improvement in rice under aerobic and normal condition. Relative water content should be given due weightage along with selection based on traits like plant height, number of tillers per plant, flag leaf area and panicle length since these traits exhibited strong positive correlation with grain yield per plot and high positive indirect effects on grain yield per via these traits indicating their true relationship with grain yield under normal condition. Number of tillers plant ${ }^{-1}$, harvest index, number of spikelets panicle ${ }^{-1}$, days to fifty per cent flowering, flag leaf area, maximum root length and proline accumulation in leaves had high to moderate positive indirect effect on grain yield plot $^{-1}$ via proline accumulation in leaves whereas days to physiological maturity, plant height, 1000 grain weight, chlorophyll content, panicle length, relative water content and peroxidase activity in leaves via proline accumulation showed high to low negative indirect effect indicated that the proline accumulation in leaves is an important trait for aerobic condition. Hence, selection based on these characters would be more effective for yield improvement in rice under aerobic condition. These results were consistent with the findings of Petchiammal and Kumar (2007) for 1000 grain weight and Kumar and Nilanjaya (2014) for relative water content.

The traits like days to fifty percent flowering, no. of spikelet panicle ${ }^{-1}$, no. of tillers plant ${ }^{-1}$ and maximum root length had positive direct effect and days to physiological maturity, plant height, panicle length, 1000 grain weight, relative water content, flag leaf area, chlorophyll content, proline accumulation and peroxidase activity had positive indirect effect via number of spikelet panicle ${ }^{-1}$, number of tillers plant ${ }^{-1}$, 


\begin{tabular}{|c|c|c|c|c|c|c|c|c|c|c|c|}
\hline No. & Character & DFF & DPM & $\mathrm{PH}$ & PL & SPP & TPP & TGW & RWC & MRL & FLA \\
\hline 1. & DFF & 0.4875 & 0.1223 & -0.2406 & -0.0112 & -0.0563 & -0.0967 & 0.0942 & 0.0049 & -0.1303 & -0.0419 \\
\hline 2. & DPM & -0.0362 & -0.1444 & -0.1384 & -0.0484 & -0.0281 & -0.0482 & -0.0446 & -0.0911 & -0.0927 & -0.0446 \\
\hline 3. & $\mathrm{PH}(\mathrm{cm})$ & 0.0856 & -0.1661 & -0.1733 & -0.1613 & -0.1513 & -0.2361 & -0.1076 & -0.1403 & -0.1578 & -0.1253 \\
\hline 4. & $\mathrm{PL}(\mathrm{cm})$ & 0.0098 & -0.1430 & -0.3967 & -0.4263 & -0.3745 & -0.2855 & -0.2589 & -0.1848 & -0.3027 & -0.2738 \\
\hline 5. & SPP & -0.0661 & 0.1114 & 0.5002 & 0.5034 & 0.5729 & 0.7051 & 0.4422 & 0.3302 & 0.4088 & 0.5206 \\
\hline 6. & TPP & -0.1365 & 0.2299 & 0.9376 & 0.4610 & 0.8473 & 0.6884 & 0.4588 & 0.4575 & 0.3893 & 0.4126 \\
\hline 7. & TGW (g) & -0.0174 & -0.0278 & -0.0560 & -0.0548 & -0.0696 & -0.0601 & -0.0902 & -0.0563 & -0.0690 & -0.0797 \\
\hline 8. & RWC (\%) & -0.0045 & -0.2823 & -0.3621 & -0.1940 & -0.4078 & -0.2973 & -0.2794 & -0.4473 & -0.2764 & -0.2800 \\
\hline 9. & $\mathrm{MRL}(\mathrm{cm})$ & -0.2225 & 0.5346 & 0.7575 & 0.5911 & 0.5939 & 0.4706 & 0.6371 & 0.5142 & 0.8323 & 0.7098 \\
\hline 10. & $\mathrm{FLA}\left(\mathrm{cm}^{2}\right)$ & -0.0094 & 0.0337 & 0.0788 & 0.0700 & 0.0990 & 0.0653 & 0.0964 & 0.0682 & 0.0930 & 0.1090 \\
\hline 11. & $\mathrm{HI}(\%)$ & 0.0631 & 0.1887 & 0.3705 & 0.2525 & 0.4391 & 0.3580 & 0.3107 & 0.2962 & 0.2957 & 0.2813 \\
\hline 12. & CC (SPAD) & -0.1231 & -0.1959 & -0.3571 & -0.3028 & -0.8929 & -0.3911 & -0.3886 & -0.2537 & -0.2816 & -0.3470 \\
\hline 13. & PRO ( $\mu \mathrm{g} \mathrm{g}^{-1}$ dry Wt.) & -0.0613 & -0.0412 & 0.0149 & -0.0013 & -0.0595 & 0.0039 & -0.0312 & -0.0097 & 0.0048 & -0.0070 \\
\hline 14. & $\begin{array}{l}\text { PERO (unit } \mathrm{g}^{-1} \text { fresh } \\
\text { Wt.) }\end{array}$ & 0.0439 & -0.0741 & -0.0872 & -0.0223 & -0.0088 & -0.0281 & -0.0194 & -0.0291 & -0.0990 & -0.0262 \\
\hline 15. & GY/P (kg) & 0.0127 & 0.1456 & 0.8480 & 0.6556 & 0.5034 & 0.8484 & 0.8195 & 0.4589 & 0.6143 & 0.8078 \\
\hline
\end{tabular}

Table 1: Continue...

\begin{tabular}{llllll}
\hline No. & Character & $\mathrm{HI}$ & $\mathrm{CC}$ & $\mathrm{PRO}$ & $\mathrm{PERO}$ \\
\hline 1. & DFF & 0.0761 & 0.1317 & -0.1798 & -0.1083 \\
2. & DPM & -0.0674 & -0.0621 & 0.0358 & -0.0541 \\
3. & PH (cm) & -0.1587 & -0.1358 & -0.0155 & -0.0765 \\
4. & PL (cm) & -0.2660 & -0.3480 & 0.0033 & -0.0480 \\
5. & SPP & 0.6219 & 0.6198 & -0.2049 & 0.0256 \\
6. & TPP & 0.6093 & 0.5909 & 0.0160 & 0.0977 \\
7. & TGW (g) & -0.0693 & -0.0769 & 0.0169 & -0.0089 \\
8. & RWC (\%) & -0.3275 & -0.5490 & 0.0261 & -0.0659 \\
9. & MRL (cm) & 0.6084 & 0.5143 & 0.0239 & 0.4167 \\
10. & FLA (cm $\left.{ }^{2}\right)$ & 0.0758 & 0.0830 & -0.0046 & 0.0144 \\
11. & HI (\%) & 0.4045 & 0.4425 & -0.0488 & 0.1393 \\
12. & CC (SPAD) & -0.4984 & -0.4557 & 0.0650 & -0.0417 \\
13. & PRO ( $\mu g g^{-1} \mathrm{dry}^{-}-0.0201$ & -0.0237 & -0.1663 & 0.0225 \\
& Wt.) & & & & \\
14. & PERO (unit g ${ }^{-1}$ & -0.0681 & -0.0181 & -0.0267 & -0.1977 \\
& fresh Wt.) & & & & \\
15. & GY/P (kg) & 0.9206 & 0.7076 & 0.1270 & 0.1152
\end{tabular}

15. GY/P (kg) $0.9206 \quad 0.7076 \quad 0.1270 \quad 0.1152$

DFF: Days to $50 \%$ flowering; DPM: Days to physiological maturity; PH: Plant height; PL: Panicle length; SPP: Spikelet panicle ${ }^{-1}$; TPP: Tillers plant ${ }^{-1}$; TGW: 1000 Grain weight; RWC: Relative water content; MRL: Maximum root length; FLA: Flag leaf area; HI: Harvest index; CC: Chlorophyll content, PRO: Proline accumulation in leaves; PERO: Peroxidase activity in leaves; GY/P: Grain yield plot ${ }^{-1}$; Residual effect: 0.3100 maximum root length and harvest index whereas, harvest index had both direct and indirect effect on grain yield per plot at genotypic level and at phenotypic level traits like days to fifty percent flowering had positive direct effect and days to physiological maturity, plant height, panicle length, 1000

Table 2: Genotypic Path coefficient analysis of fifteen characters on grain yield in rice under normal condition

\begin{tabular}{llllll}
\hline No. & Character & DFF & DPM & PH & PH \\
\hline 1. & DFF & -0.0177 & -0.0022 & -0.0008 & -0.0008 \\
2. & DPM & -0.0809 & -0.6479 & -0.6621 & -0.6621 \\
3. & PH (cm) & 0.0070 & 0.1533 & 0.1500 & 0.1500 \\
4. & PL (cm) & -0.0274 & 0.1595 & 0.2587 & 0.2587 \\
5. & SPP & -0.2722 & -0.4922 & -0.8392 & -0.8392 \\
6. & TPP & 0.0619 & 0.4480 & 0.6253 & 0.6253 \\
7. & TGW (g) & 0.0809 & 0.3866 & 0.4252 & 0.4252 \\
8. & RWC (\%) & 0.2413 & 0.7422 & 0.9469 & 0.9469 \\
9. & MRL (cm) & 0.2042 & 0.9212 & 1.3292 & 1.3292 \\
10. & FLA (cm $\left.{ }^{2}\right)$ & -0.0375 & -0.1937 & -0.5994 & -0.5994 \\
11. & HI (\%) & 0.0033 & -0.0016 & -0.0098 & -0.0098 \\
12. & CC (SPAD) & -0.1260 & -0.5125 & -1.0012 & -1.0012 \\
13. & PRO ( $\mu g$ g g dry & 0.0731 & -0.0015 & -0.0102 & -0.0102 \\
& Wt.) & & & & \\
14. & PERO (unit g ${ }^{-1}$ & -0.0489 & -0.0432 & -0.0041 & -0.0041 \\
& fresh Wt.) & & & & \\
15. & GY/P (kg) & 0.0611 & 0.9158 & 0.6084 & \\
\hline
\end{tabular}
Continue... 


\begin{tabular}{|c|c|c|c|c|c|c|c|c|c|c|c|c|}
\hline No. & Character & $\mathrm{PL}$ & SPP & TPP & TGW & RWC & MRL & FLA & $\mathrm{HI}$ & CC & PRO & PERO \\
\hline 1. & DFF & 0.0018 & -0.0089 & -0.0019 & -0.0032 & -0.0044 & -0.0031 & -0.0019 & 0.0031 & -0.0021 & 0.0035 & -0.0060 \\
\hline 2. & DPM & -0.3798 & -0.5872 & -0.4972 & -0.5520 & -0.4910 & -0.5060 & -0.3510 & -0.0535 & -0.3078 & -0.0027 & -0.1954 \\
\hline 3. & $\mathrm{PH}(\mathrm{cm})$ & 0.1426 & 0.1627 & 0.1606 & 0.1405 & 0.1450 & 0.1690 & 0.1466 & 0.0762 & 0.1392 & 0.0042 & 0.0043 \\
\hline 4. & $\mathrm{PL}(\mathrm{cm})$ & 0.2720 & 0.2224 & 0.2077 & 0.1567 & 0.2120 & 0.2208 & 0.2033 & 0.0715 & 0.2107 & 0.0344 & -0.0142 \\
\hline 5. & SPP & -0.4440 & -0.5431 & -0.5064 & -0.4342 & -0.6015 & -0.5517 & -0.4946 & -0.2570 & -0.5029 & -0.0159 & -0.1008 \\
\hline 6. & TPP & 0.4457 & 0.5443 & 0.5838 & 0.4653 & 0.4515 & 0.5894 & 0.4325 & 0.3252 & 0.5016 & 0.0593 & 0.1449 \\
\hline 7. & TGW (g) & 0.2614 & 0.3627 & 0.3616 & 0.4538 & 0.3284 & 0.3681 & 0.3425 & 0.1930 & 0.3037 & -0.0054 & 0.1531 \\
\hline 8. & RWC (\%) & 0.7634 & 1.0848 & 0.7575 & 0.7089 & 0.9795 & 0.8415 & 0.7131 & 0.5829 & 0.7206 & 0.1232 & 0.1930 \\
\hline 9. & $\mathrm{MRL}(\mathrm{cm})$ & 0.9573 & 1.1981 & 1.1908 & 0.9570 & 1.0133 & 1.1796 & 1.0621 & 0.3323 & 1.1245 & -0.1412 & 0.1918 \\
\hline 10. & FLA (cI & -0 & -0.3256 & -0.2649 & -0.2699 & 603 & -0.3219 & -0.3575 & -0.1282 & -0.2837 & 0.0795 & -0.0493 \\
\hline 11. & $\mathrm{HI}(\%)$ & -0. & -0.35 & -0.0107 & -0.0 & 15 & -0.0 & -0.0069 & -0.0193 & -0.0084 & -0.0078 & -0.0006 \\
\hline 12. & $\mathrm{CC}(\mathrm{SPAD})$ & -0.8354 & -0.9989 & -0.9268 & -0.7221 & -0.7936 & -1.0284 & -0.8559 & -0.4692 & -1.0787 & 0.2687 & -0.0309 \\
\hline 13. & $\begin{array}{l}\text { PRO }\left(\mu g^{-1}\right. \\
\text { dry Wt.) }\end{array}$ & -0.0464 & -0.1198 & -0.0372 & 0.0044 & -0.0461 & 0.0439 & 0.0816 & -0.1488 & 0.0709 & -0.3067 & -0.0326 \\
\hline 14. & $\begin{array}{l}\text { PERO (unit } \\
\mathrm{g}^{-1} \text { fresh } \\
\text { Wt.) }\end{array}$ & 0.0075 & -0.0275 & -0.0356 & -0.0483 & -0.0282 & -0.0233 & -0.0198 & -0.0047 & -0.0041 & -0.0127 & -0.1433 \\
\hline 15. & $\mathrm{GY} / \mathrm{P}(\mathrm{kg})$ & 0.8739 & 0.6049 & 0.9813 & 0.8486 & 0.8932 & 0.9725 & 0.8941 & 0.5035 & 0.8835 & 0.0804 & 0.1139 \\
\hline
\end{tabular}

Residual Effect $=0.2704$

grain weight, relative water content, proline accumulation in leaves, peroxidase activity, no. of tillers plant ${ }^{-1}$ and maximum root length had positive indirect effect via no. of spikelet, 1000 grain weight, maximum root length whereas, no. of spikelet panicle ${ }^{-1}$, flag leaf area and chlorophyll content had both direct and indirect effect on grain yield plot $^{-1}$ under aerobic condition.

The traits like relative water content and maximum root length

\begin{tabular}{|c|c|c|c|c|c|c|c|c|c|c|c|}
\hline No. & Character & DFF & DPM & $\mathrm{PH}$ & $\mathrm{PL}$ & SPP & TPP & TGW & RWC & MRL & FLA \\
\hline 1. & DFF & -0.0867 & -0.0240 & 0.0042 & -0.0010 & 0.0050 & 0.0003 & -0.0144 & -0.0015 & 0.0146 & 0.0047 \\
\hline 2. & DPM & 0.0139 & 0.0502 & -0.0007 & 0.0091 & 0.0059 & 0.0070 & 0.0138 & 0.0183 & 0.0145 & 0.0098 \\
\hline 3. & $\mathrm{PH}(\mathrm{cm})$ & 0.0012 & 0.0004 & -0.0250 & -0.0072 & -0.0078 & -0.0116 & -0.0059 & -0.0095 & -0.0106 & -0.0065 \\
\hline 4. & $\mathrm{PL}(\mathrm{cm})$ & -0.0012 & -0.0189 & -0.0304 & -0.1046 & -0.0354 & -0.0366 & -0.0570 & -0.0369 & -0.0513 & -0.0547 \\
\hline 5. & SPP & -0.0186 & 0.0378 & 0.1011 & 0.1092 & 0.3222 & 0.0661 & 0.1339 & 0.1163 & 0.1248 & 0.1253 \\
\hline 6. & TPP & -0.0003 & 0.0130 & 0.0432 & 0.0324 & 0.0190 & 0.0927 & 0.0343 & 0.0325 & 0.0462 & 0.0322 \\
\hline 7. & TGW (g) & 0.0263 & 0.0435 & 0.0378 & 0.0864 & 0.0659 & 0.0587 & 0.1585 & 0.0859 & 0.0952 & 0.1084 \\
\hline 8. & RWC (\%) & -0.0031 & -0.0650 & -0.0678 & -0.0628 & -0.0643 & -0.0625 & -0.0966 & -0.1782 & -0.0989 & -0.0986 \\
\hline 9. & $\operatorname{MRL}(\mathrm{cm})$ & 0.0191 & -0.0329 & -0.0483 & -0.0557 & -0.0440 & -0.0566 & -0.0682 & -0.0630 & -0.1135 & -0.0766 \\
\hline 10. & $\mathrm{FLA}(\mathrm{cm} 2)$ & -0.0146 & 0.0527 & 0.0705 & 0.1412 & 0.1050 & 0.0937 & 0.1847 & 0.1495 & 0.1823 & 0.2700 \\
\hline 11. & HI (\%) & 0.0048 & 0.0435 & 0.0708 & 0.1017 & 0.0858 & 0.0814 & 0.1262 & 0.1104 & 0.1047 & 0.1132 \\
\hline 12. & CC (SPAD) & 0.0579 & 0.0458 & 0.1151 & 0.1346 & 0.1610 & 0.1136 & 0.1714 & 0.1317 & 0.1236 & 0.1513 \\
\hline 13. & $\begin{array}{l}\text { PRO ( } \mu \mathrm{g} \mathrm{g}^{-1} \\
\text { dry Wt.) }\end{array}$ & 0.0013 & 0.0005 & -0.0003 & 0.0001 & 0.0004 & 0.0002 & 0.0007 & 0.0003 & 0.0001 & 0.0003 \\
\hline 14. & $\begin{array}{l}\text { PERO (unit g }{ }^{-1} \\
\text { fresh Wt.) }\end{array}$ & -0.0035 & 0.0050 & 0.0051 & 0.0021 & 0.0006 & 0.0020 & 0.0020 & 0.0034 & 0.0105 & 0.0027 \\
\hline 15. & $\mathrm{GY} / \mathrm{P}(\mathrm{kg})$ & -0.0037 & 0.1514 & $0.2754^{*}$ & $0.3855^{* *}$ & $0.6193^{* *}$ & $0.3484^{* *}$ & $0.5833^{* *}$ & $0.3592^{* *}$ & $0.4420^{* *}$ & $0.5814^{* *}$ \\
\hline
\end{tabular}




\begin{tabular}{llcccc}
\hline \multicolumn{6}{l}{ Table 3: Continue... } \\
\hline No. & Character & HI & CC & PRO & PERO \\
\hline 1. & DFF & -0.0020 & -0.0165 & 0.0235 & 0.0128 \\
2. & DPM & 0.0105 & 0.0075 & 0.0058 & 0.0106 \\
3. & PH (cm) & -0.0085 & -0.0094 & 0.0017 & -0.0054 \\
4. & PL (cm) & -0.0510 & -0.0463 & 0.0014 & -0.0093 \\
5. & SPP & 0.1325 & 0.1705 & 0.0297 & 0.0080 \\
6. & TPP & 0.0362 & 0.0346 & 0.0038 & 0.0078 \\
7. & TGW (g) & 0.0960 & 0.0893 & 0.0221 & 0.0131 \\
8. & RWC (\%) & -0.0943 & -0.0772 & 0.0103 & -0.0258 \\
9. & MRL (cm) & -0.0570 & -0.0461 & 0.0008 & -0.0504 \\
10. & FLA (cm $\left.{ }^{2}\right)$ & 0.1466 & 0.1343 & 0.0153 & 0.0307 \\
11. & HI (\%) & 0.2085 & 0.1299 & 0.0214 & 0.0523 \\
12. & CC (SPAD) & 0.1895 & 0.3043 & 0.0443 & 0.0190 \\
13. & PRO ( $\mu$ g g ${ }^{-1}$ & 0.0005 & 0.0007 & 0.0047 & -0.0006 \\
& dry Wt.) & & & & \\
14. & PERO (unit & 0.0059 & 0.0015 & 0.0029 & 0.0237 \\
& g $^{-1}$ fresh Wt.) & & & & \\
15. & GY/P (kg) & $0.6133^{* *}$ & $0.6771^{* *}$ & 0.1112 & 0.0866 \\
\hline
\end{tabular}

Residual effect $=0.5794$

Table 4: Phenotypic Path coefficient analysis of fifteen characters on grain yield in rice under normal condition

\begin{tabular}{|c|c|c|c|c|c|}
\hline No. & Character & DFF & DPM & $\mathrm{PH}$ & $\mathrm{PL}$ \\
\hline 1. & DFF & 0.0485 & 0.0116 & 0.0012 & -0.0058 \\
\hline 2. & DPM & 0.0241 & 0.1013 & 0.0361 & 0.0376 \\
\hline 3. & $\mathrm{PH}(\mathrm{cm})$ & 0.0026 & 0.0368 & 0.1034 & 0.0660 \\
\hline 4. & $\mathrm{PL}(\mathrm{cm})$ & -0.0186 & 0.0576 & 0.0991 & 0.1553 \\
\hline 5. & SPP & 0.0090 & 0.0198 & 0.0306 & 0.0232 \\
\hline 6. & TPP & -0.0005 & 0.0432 & 0.0485 & 0.0624 \\
\hline 7. & TGW (g) & 0.0093 & 0.0875 & 0.0928 & 0.0885 \\
\hline 8. & RWC (\%) & 0.0116 & 0.0457 & 0.0526 & 0.0613 \\
\hline 9. & MRL (cm) & 0.0171 & 0.0742 & 0.0883 & 0.1007 \\
\hline 10. & $\mathrm{FLA}\left(\mathrm{cm}^{2}\right)$ & 0.0047 & 0.0439 & 0.0652 & 0.0747 \\
\hline 11. & $\mathrm{HI}(\%)$ & 0.0011 & -0.0019 & -0.0051 & -0.0044 \\
\hline 12. & $\mathrm{CC}(\mathrm{SPAD})$ & -0.0011 & 0.0191 & 0.0210 & 0.0289 \\
\hline 13. & $\begin{array}{l}\text { PRO ( } \mu \mathrm{g} \mathrm{g}^{-1} \\
\text { dry Wt.) }\end{array}$ & -0.0012 & 0.0000 & 0.0002 & 0.0008 \\
\hline 14. & $\begin{array}{l}\text { PERO (unit } \\
g^{-1} \text { fresh } \\
\text { Wt.) }\end{array}$ & -0.0143 & -0.0134 & -0.0014 & 0.0036 \\
\hline 15. & $\mathrm{GY} / \mathrm{P}(\mathrm{kg})$ & 0.0925 & $0.5256^{* *}$ & $0.6327^{* *}$ & $0.6927^{* *}$ \\
\hline
\end{tabular}

Table 4: Continue...

\begin{tabular}{|c|c|c|c|c|c|c|c|c|c|c|c|}
\hline No. & Character & SPP & TPP & TGW & RWC & MRL & FLA & $\mathrm{HI}$ & CC & PRO & PERO \\
\hline 1. & DFF & 0.0077 & -0.0002 & 0.0027 & 0.0064 & 0.0051 & 0.0021 & -0.0027 & -0.0010 & -0.0076 & 0.0115 \\
\hline 2. & DPM & 0.0353 & 0.0369 & 0.0519 & 0.0530 & 0.0456 & 0.0408 & 0.0095 & 0.0355 & 0.0006 & 0.0225 \\
\hline 3. & $\mathrm{PH}(\mathrm{cm})$ & 0.0556 & 0.0423 & 0.0562 & 0.0623 & 0.0554 & 0.0619 & 0.0265 & 0.0398 & 0.0033 & 0.0023 \\
\hline 4. & $\mathrm{PL}(\mathrm{cm})$ & 0.0633 & 0.0817 & 0.0806 & 0.1091 & 0.0949 & 0.1065 & 0.0348 & 0.0825 & 0.0156 & -0.0093 \\
\hline 5. & SPP & 0.0569 & 0.0124 & 0.0198 & 0.0326 & 0.0286 & 0.0283 & 0.0240 & 0.0228 & 0.0073 & 0.0070 \\
\hline 6. & TPP & 0.0259 & 0.1187 & 0.0642 & 0.0654 & 0.0716 & 0.0649 & 0.0294 & 0.0584 & 0.0097 & 0.0215 \\
\hline 7. & TGW (g) & 0.0594 & 0.0924 & 0.1707 & 0.1052 & 0.1017 & 0.1152 & 0.0487 & 0.0811 & 0.0055 & 0.0490 \\
\hline 8. & RWC (\%) & 0.0500 & 0.0482 & 0.0538 & 0.0873 & 0.0575 & 0.0607 & 0.0308 & 0.0502 & 0.0098 & 0.0166 \\
\hline 9. & $\mathrm{MRL}(\mathrm{cm})$ & 0.0828 & 0.0994 & 0.0982 & 0.1085 & 0.1647 & 0.1190 & 0.0455 & 0.1032 & -0.0043 & 0.0227 \\
\hline 10. & $\mathrm{FLA}\left(\mathrm{cm}^{2}\right)$ & 0.0541 & 0.0595 & 0.0735 & 0.0756 & 0.0786 & 0.1089 & 0.0282 & 0.0698 & -0.0204 & 0.0153 \\
\hline 11. & $\mathrm{HI}(\%)$ & -0.0084 & -0.0049 & -0.0057 & -0.0070 & -0.0055 & -0.0051 & -0.0198 & -0.0052 & -0.0053 & -0.0002 \\
\hline 12. & CC (SPAD) & 0.0218 & 0.0268 & 0.0258 & 0.0313 & 0.0341 & 0.0349 & 0.0143 & 0.0544 & -0.0090 & 0.0018 \\
\hline 13. & $\begin{array}{l}\text { PRO ( } \mu \mathrm{g} \mathrm{g}^{-1} \\
\text { dry Wt.) }\end{array}$ & 0.0010 & 0.0006 & 0.0003 & 0.0009 & -0.0002 & -0.0015 & 0.0021 & -0.0013 & 0.0078 & 0.0005 \\
\hline 14. & $\begin{array}{l}\text { PERO (unit g-1 } \\
\text { fresh Wt.) }\end{array}$ & -0.0074 & -0.0109 & -0.0173 & -0.0115 & -0.0083 & -0.0085 & -0.0007 & -0.0020 & -0.0042 & -0.0602 \\
\hline 15. & $\mathrm{GY} / \mathrm{P}(\mathrm{kg})$ & $0.4981^{* *}$ & $0.6028^{* *}$ & $0.6746^{* *}$ & $0.7192^{* *}$ & $0.7240^{* *}$ & $0.7280^{* *}$ & $0.2706^{*}$ & $0.5883^{* *}$ & 0.0087 & 0.1010 \\
\hline
\end{tabular}

Residual effect $=0.5218$

had positive but proline accumulation had negative direct effect and days to physiological maturity, plant height, panicle length, flag leaf area, chlorophyll content, harvest index, days

to $50 \%$ flowering, no. of spikelet panicle ${ }^{-1}$, and peroxidase activity had positive indirect effect via no. of spikelet, 1000 grain weight, no. of tillers plant ${ }^{-1}$, harvest index, flag leaf 
area and chlorophyll content whereas, no. of tillers plant ${ }^{-1}$ and 1000 grain weight had both direct and indirect effect on grain yield plot $^{-1}$ at genotypic level and at phenotypic level traits like proline accumulation in leaves, had positive direct effect but days to physiological maturity, plant height, panicle length, 1000 grain weight, relative water content, peroxidase activity, no. of tillers plant ${ }^{-1}$, maximum root length, no. of spikelet panicle ${ }^{-1}$, flag leaf area and chlorophyll content had positive indirect effect via plant height, panicle length, no. of tillers plant ${ }^{-1}, 1000$ grain weight, relative water content, maximum root length and flag leaf area whereas, days to $50 \%$ flowering had both direct and indirect effect on grain yield per plot under normal condition. Hence, selection based on this character will be more effective for yield improvement in rice under aerobic and normal condition.

\section{Conclusion}

Path analysis revealed at genotypic level that under normal condition traits like proline accumulation in leaves $(-0.31)$ had high negative direct effect and traits like days to $50 \%$ flowering, number of spikelets panicle ${ }^{-1}$, number of tillers plant $^{-1}$, relative water content in flag leaf, and maximum root length had high positive direct effect. The genotype RAU1417-2-1-5-7-7 were identified as promising parents for their further utilization in hybridization programme in order to develop genotypes suitable for both normal and aerobic condition when these important plant traits must be taken into consideration when any breeding program for higher yield in rice under aerobic and normal conditions is to be planned.

\section{Acknowledgement}

Authors are thankful to Dr. Rajendra Prasad Central Agricultural University, Pusa, Samastipur for providing genotypes of rice suitable for aerobic and normal condition and all the members of Department of Plant Breeding and Genetics for their encouragement, support and providing necessary facilities.

\section{References}

Agahi, K., Fotokian, M.H., Farshadfar, E., 2007. Path coefficient analysis for some yield related traits in rice genotypes. Asian Journal of Plant Sciences 6(3), 513-517.
Barr, H.D., Weatherley, P.E., 1962. A re-examination of the relative turgidity technique for estimating water deficit in leaves. Australian Journal of Biological Science 15, 413-428.

Bates, L., Waldren, R.P., Teare, I.D., 1973. Rapid determination of free proline for water-stress studies. Plant and Soil 39, 205-207.

Dewey, D.R., Lu, K.H., 1959. Correlation and path coefficient analysis of components of crested wheat grass for seed production. Agronomy Journal 51, 515-518.

Donald, C.M., 1962. In search of yield. Journal of the Australian Institute of Agricultural Sciences 28, 172-178.

Kumar, C., Nilanjaya., 2014. Correlation and Path coefficient analysis of yield components in aerobic rice (Oryza sativa L.). The Bioscan 9(2), 907-913.

Milligan, S.B., Gravois, K.A., Bischoff, K.P., Martin, F.A., 1990. Crop effects on genetic relationships among sugarcane traits. Crop Science 30, 927-931.

Mustafa, M.A., Elsheikh, M.A.Y., 2007. Path coefficient analysis for yield and its components in rice. African crop Sciences Journal 15(4), 183-189.

Nagaraju, C., Sekhar, M.R., Reddy, K.H., Sudhakar, P., 2013. Correlation between traits and path analysis coefficient for grain yield and other components in rice (Oryza sativa L.) genotypes. International Journal of Applied Biology and Pharmaceutical Technology 4(3), 137-142.

Palmiano, E.P., Juliano, B.C., 1973. Changes in the activity of some hydrolases, peroxidase and catalase in rice seed during germination. Plant Physiology 52, 274-277.

Patel, J.R., Saiyad, M.R., Prajapati, K.N., Patel, R.A., Bhavani R.T., 2014. Genetic variability and character association studies in rainfed upland rice (Oryza sativa L.). Electronic Journal of Plant Breeding 5(3), 531-537.

Petchiammal, K.I., Kumar, C.R.A., 2007. Association analysis for yield and related traits in rice. International journal of Plant Sciences Muzaffarpurnagar 22(2), 97-100.

Ullah, M.Z., Bashar, M.K., Bhuiyan, M.R., Khalequzzaman, M., Hassan, M.J., 2011. Inter relationship and cause and effect analysis among morpho-physiological traits in rice. African Journal of Biotechnology 9, 7853-7856.

Wright, S., 1921. Correlation and causation. Journal of Agricultural Research 20, 557-585. 